\title{
Ermittlung von Berechnungsverfahren für den erzielbaren Volumenstrom von stark geneigten Schneckenförderern
}

\author{
Prof. DR.-ING. DiPL.-WI.-ING. WiLlibALD A. GÜNTHNER, \\ DIPL.-ING. STEFAN RAKITSCH \\ LEHRSTUHL FÜR FÖRDERTECHNIK MATERIALFLUSS LOGISTIK, TECHNISCHE UNIVERSITÄT MÜNCHEN \\ DiPL.-STAT. PAUL SCHMIDT \\ STEINBEIS-FORSCHUNGSZENTRUM ANCORESTATISTICS
}

\section{Zusammenfassung}

Die Dimensionierung und Auslegung von Schneckenförderern zur stark geneigten Förderung von Schüttgut beruht heute noch oft auf Erfahrungswerten der Anlagenhersteller. Um eine effiziente Auslegung auf Basis gesicherter Berechnungsverfahren zu gewährleisten, läuft am Lehrstuhl für Fördertechnik Materialfluss Logistik (fml) derzeit ein von der Deutschen Forschungsgemeinschaft (DFG) gefördertes Projekt im Rahmen dessen die Schüttgutförderung in geneigten Schneckenförderern untersucht wird. Entscheidender Bestandteil der Untersuchung ist dabei die Vorhersage des Förderverhaltens im Schneckenförderer und damit die Bestimmung des erzielbaren Volumenstroms in Abhängigkeit der vorliegenden Geometrie- und Betriebsparameter. Um dies zu gewährleisten werden Daten für den erzielten Volumenstrom für eine große Anzahl verschiedener Parameterkombinationen gewonnen und anschließend statistisch ausgewertet. Durch eine Regressionsanalyse wird schließlich auf einen berechenbaren Zusammenhang geschlossen.

\section{Abstract}

The dimensioning and sizing of heavy inclined screw conveyors for bulk materials is today often based on the experience of the manufacturers. To assure an efficient sizing based on trusted calculation methods the Institute of Materials Handling Material Flow Logistics (fml) is working on a research project, funded by the Deutsche Forschungsgemeinschaft (DFG), which is aimed at analyzing inclined screw conveyors. Determining element of the project is the forecast of the conveying character inside the Screw conveyor and therewith the designation of the achievable volume flows as a function of the geometry and operating parameters. In order to achieve this data for the achievable volume flow for numerousness different parameter combinations are gained and statistically evaluated. By the use of regression analyses a computable interrelationship is finally developed.

\section{Einleitung}

Die Vorteile, wie der einfache und robuste Aufbau, die niedrigen Anlagen- und Wartungskosten, die geringe Störanfälligkeit und im Besonderen die staubdichte Ausführung, führen häufig zum Einsatz von Schneckenförderern für den stetigen Schüttguttransport. Konventionelle Schneckenförderer sind für den Transport staubförmiger, körniger und leicht feuchter Schüttgüter geeignet. Stark geneigte Schneckenförderer finden beispielsweise beim Massengutumschlag, siehe Abbildung 1, oder beim Siloaustrag Anwendung.

Die Anforderungen hinsichtlich Zuverlässigkeit, Leistungsfähigkeit und Wirtschaftlichkeit aber auch bezüglich der Energieeffizienz und des Umweltschutzes für Schneckenförderer sind in den letzten Jahren deutlich gestiegen. Schneckenförderer müssen deshalb, wie andere Stetigförderer für Schüttgut auch, zuverlässig ausgelegt werden, um Ausfallzeiten oder Überdimensionierung zu vermeiden. Nachdem in den letzten Jahren durch Forschungsarbeiten am Lehrstuhl für Fördertechnik Materialfluss Logistik bereits solche Dimensionierungs- und Auslegungsverfahren für horizontal bis leicht geneigte bzw. vertikale Schneckenförderer erarbeitet wurden, wird durch dieses Projekt nun die Lücke bei den stark geneigten Förderern geschlossen. 


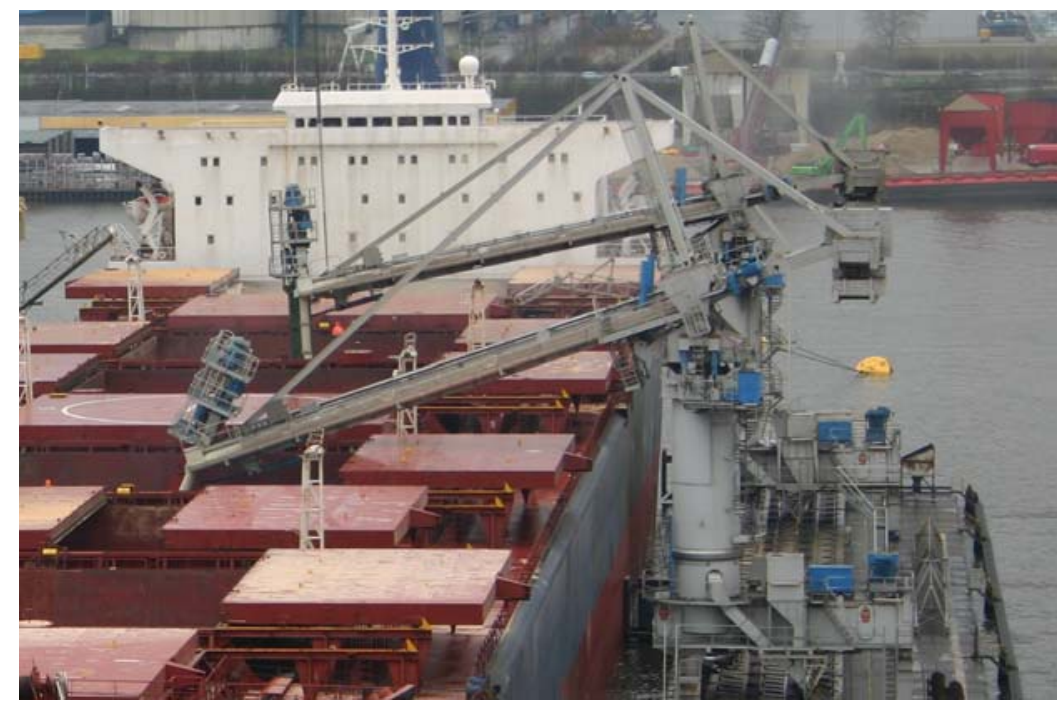

Abbildung 1: Massengutumschlag mit Hilfe von Schneckenförderern

Schneckenförderer bestehen im Wesentlichen aus einem Fördertrog oder -rohr und der Förderschnecke, bestehend aus Schneckenwelle und -wendel. Der Guttransport beruht darauf, dass das Schüttgut, welches durch Schwerkraft und/oder Zentripetalkraft ans Rohr gedrückt wird, durch die Schneckenwendel vorwärts geschoben wird. Liegt das Schüttgut nur durch die Schwerkraft auf dem Rohr spricht man von einer translatorischen Förderung - das Schüttgut wird ohne nennenswerte Umwälzung translatorisch vorwärts bewegt - wohingegen man beim Anpressen des Schüttguts ausschließlich infolge einer Zentripetalkraft von einer rotatorischen Bewegung ein Großteil der Bewegungsenergie wird in Rotation umgesetzt - spricht. Bei der horizontalen und leicht geneigten Förderung kann die Gutbewegung unter bestimmten Voraussetzungen als rein translatorisch betrachtet werden. Beim Übergang in den stärker geneigten Bereich wird diese translatorische Gutbewegung von einem rotatorischen Anteil überlagert bis beim Extremfall der vertikalen Förderung nur noch die rotatorische Gutbewegung vorherrscht. Ein translatorischer Anteil ist ab einer gewissen Schneckenneigung aufgrund des Schwerkrafteinflusses nicht mehr möglich. Entscheidenden Einfluss auf die Art der Förderung haben die Schneckenneigung $\beta$ und die Schneckendrehzahl $n$ des Schneckenförderers [Vol-2000]. Die qualitative Einteilung des Förderverhaltens in die rein translatorische Förderung (Bereich I), die rein rotatorische Förderung (Bereich III) und den Bereich II mit Überlagerung der vorgenannten Formen ist in Abbildung 2 dargestellt. Im Bereich III und teilweise im Bereich II ist eine Förderung nur möglich, wenn die Schneckenwendel eine gewisse Mindestdrehzahl aufweist. Diese Mindestdrehzahl ist nötig, damit das Schüttgut durch die Zentripetalkraft am Förderrohr gehalten wird und nicht nach unten abgleitet.

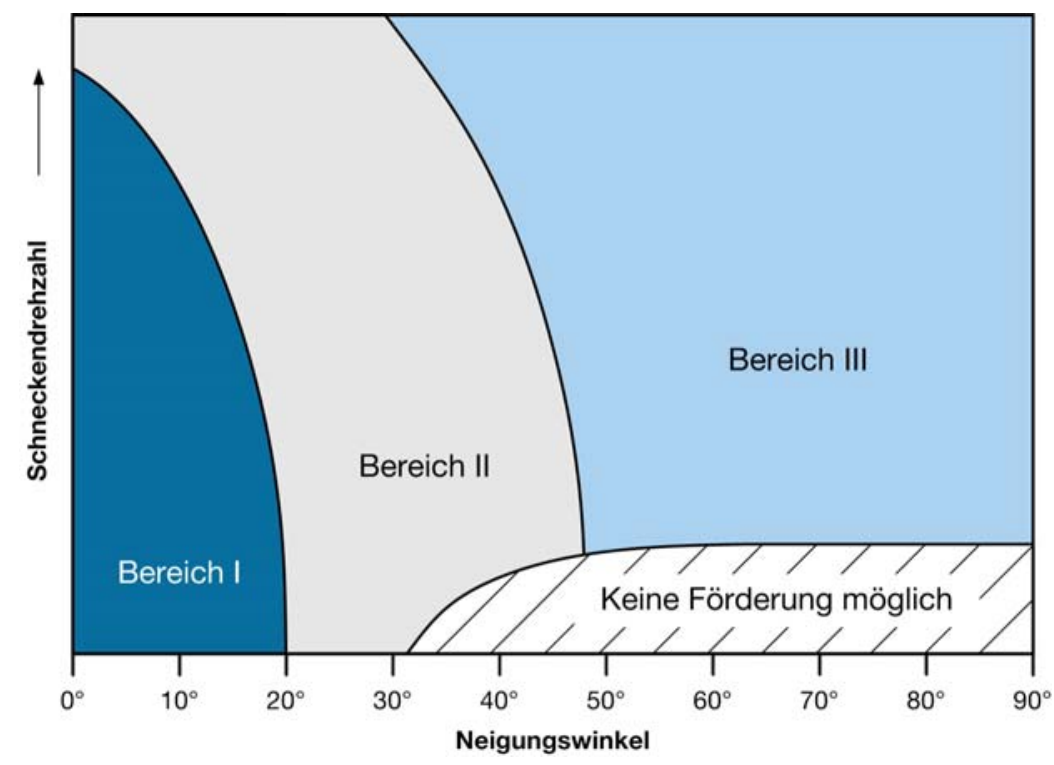

Abbildung 2: Arten der Förderung im Schneckenförderer nach Schneckendrehzahl und Neigung [Vol-2000] 


\section{Stand der Forschung}

Am Lehrstuhl für Fördertechnik Materialfluss Logistik wurden in den letzten Jahren bereits zahlreiche Arbeiten durchgeführt, die sich der Beschreibung von Schneckenförderern und der Entwicklung von Dimensionierungsund Auslegungsvorschriften widmeten. Gerade die letzten Arbeiten ([Gün-2002], [Gün-2006]) hatten die Schaffung von praxistauglichen Verfahren, die für den Anwender leicht zu beherrschen sind, zum Ziel. Für die horizontalen bis leicht geneigten Schneckenförderer entstand so ein Verfahren, welches stark an die DIN 15262 [DIN 15262] angelehnt ist. Die Berechnung kann der Arbeit von Blomeyer [Blo-2008] entnommen werden. In den genannten Arbeiten wurde ebenfalls ein Dimensionierungs- und Auslegungsverfahren für vertikal betriebene Schneckenförderer entwickelt. Diese Verfahren wurden als teilweise grafische Verfahren realisiert. Die genauen Berechnungsformeln sowie weitere Diagramme zur Volumenstrombestimmung können der Arbeit von Rong [Ron-2004] entnommen werden.

Ebenfalls wurden bereits Untersuchungen an stark geneigten Schneckenförderern durchgeführt. Aufbauend auf die Arbeit von Gabler [Gab-1981] wurden dazu von Vollmann [Vol-2000] immer feinere Berechnungsalgorithmen und Programme entwickelt, mit denen es möglich ist den Volumenstrom und die Antriebsleistung von stark geneigten Schneckenförderern zu berechnen. Grundlage ist die analytische Betrachtung der Spannungsverhältnisse im Inneren des Schüttguts und an den Berührungspunkten mit dem Schneckenförderer. Das Schüttgut im Schneckenförderer wird dazu in Segmente unterteilt, für die einzeln die Bewegungsgleichungen gelöst werden. Aus der Aufsummierung der einzelnen Geschwindigkeiten und Reibkräfte kann schließlich der Volumenstrom berechnet werden. Obwohl diese Betrachtungen die Förderung in geneigten Schneckenförderern gut beschreiben, genügen sie nicht dem Ansatz eines einfachen und praxistauglichen Berechnungsverfahrens. Der Grund liegt in der nötigen Komplexität des analytischen Modells, das nicht durch einen geschlossen lösbaren Gleichungssatz beschrieben werden kann. Ein weiterer Nachteil besteht darin, dass nur der Bereich mit rein rotatorischer Förderung analytisch beschrieben werden kann und das Verfahren daher nur für diesen Bereich gültig ist. Dennoch liefert das Verfahren wertvolle Aussagen zum Förderverhalten und den Einflüssen der verschiedenen Parameter und bildet daher eine wichtige Grundlage für die vorliegende Untersuchung.

\section{Aufgabenstellung}

Ziel der Untersuchung ist die Entwicklung eines einfach anwendbaren und sicheren Dimensionierungsverfahrens für stark geneigte Schneckenförderer für Schüttgut unter Berücksichtigung von Konstruktions-, Betriebs- und Schüttgutparametern. Da die Grenzen zwischen den Bereichen mit rein rotatorischer Förderung und der gemischten Förderung sowie das Verhalten des Schüttguts im Bereich gemischter Förderung analytisch nicht beschreibbar sind, konnten sie deshalb bisher noch nicht vollständig beschrieben werden [Vol-2000]. Im Rahmen dieser Untersuchung wird deshalb ein semi-empirisches Verfahren angewendet, um den erzielbaren Volumenstrom im geneigten Schneckenförderer bestimmen zu können. Grundlage für ein semi-empirisches Verfahren ist ein analytischer Grundansatz, der um empirische Kenngrößen erweitert wird. Diese bilden die analytisch nicht erfassbaren Einflüsse in Abhängigkeit der bekannten Parameter ab. Damit ist es auch möglich die Berechnungsverfahren so zu gestalten, dass sie an das Vorgehen von bekannten Normen und Richtlinien, wie der DIN 15262 [DIN 15262] oder der VDI 2330 [VDI 2330], erinnern und damit eine hohe Akzeptanz unter den potentiellen Anwendern zu erwarten ist.

\section{Berechnungsmodell}

Die Berechnung des geförderten Volumenstroms folgt dem prinzipiellen Vorgehen zur Berechnung des Volumenstroms für Stetigförderer für Schüttgut und wird auch in den bisherigen Berechnungsansätzen für Schneckenförderer angewendet ([DIN 15262], [Gün-2002], [Gün-2006]). Demnach berechnet sich der geförderte Volumenstrom eines Schneckenförderers gemäß der aus der Strömungsmechanik bekannten Kontinuitätsgleichung als Produkt von durchschnittlicher Geschwindigkeitskomponente in Förderrichtung und der orthogonal dazu stehenden durchflossenen Fläche.

Als durchflossene Fläche $A$ wird der Kreisring, den der Schneckendurchmesser $D$ und der Wellendurchmesser $d$ beschreiben, verwendet. Da der Schneckenförderer nicht komplett gefüllt ist, reduziert sich diese Fläche um den Füllungsgrad $\varphi$ und berechnet sich nach

$$
A=\pi \cdot \frac{1}{4}\left(D^{2}-d^{2}\right) \cdot \varphi
$$


Während sich die Fördergeschwindigkeit beim horizontalen Schneckenförderer nur aus dem Produkt von Schneckenganghöhe $S$ und Schneckendrehzahl $n$ berechnet, muss beim geneigten Schneckenförderer die Rotation des Schüttguts mit beachtet werden. Gemäß der Arbeit von Böttcher [Böt-1963] erfolgt die Berechnung der durchschnittlichen axialen Fördergeschwindigkeit $v_{a x}$ nach

$$
v_{a x}=\frac{S}{2 \pi} \cdot\left(\omega_{S}-\omega_{G}\right)
$$

aus den Winkelgeschwindigkeiten der Schnecke $\omega_{S}$ und des Förderguts $\omega_{G}$ sowie der Schneckenganghöhe $S$. Die Differenz der Winkelgeschwindigkeiten von Schnecke und Fördergut entspricht der Relativwinkelgeschwindigkeit zwischen Fördergut und Schneckenwendel in Förderrichtung, die zur Förderung notwendig ist. Die Winkelgeschwindigkeit der Schnecke muss daher größer als die Gutwinkelgeschwindigkeit sein, damit die Geschwindigkeitsdifferenz positiv ist. Um dem verfolgten Ansatz eines einfachen und praxistauglichen Dimensionierungsverfahrens gerecht zu werden, wird diese Differenz zwischen Schnecken- und Gutwinkelgeschwindigkeit als Anteil der Winkelgeschwindigkeit der Schnecke dargestellt und gemäß

$$
\omega_{S}-\omega_{G}=\omega_{S} \cdot \zeta
$$

berechnet, wobei $\zeta$ einen empirischen Geschwindigkeitsbeiwert darstellt, der im Intervall $0 \leq \zeta \leq 1$ liegt. Die Grenzen dieses Beiwerts sind anschaulich zu erklären: Einerseits verschwindet für $\zeta=0$ die Relativwinkelgeschwindigkeit und es findet keine Förderung mehr statt. Dieser Fall stellt das Unterschreiten der Mindestdrehzahl dar. Andererseits wird für $\zeta=1$ die Relativwinkelgeschwindigkeit gleich der Winkelgeschwindigkeit der Schnecke und nimmt damit den maximal möglichen Wert an. Mit diesen Überlegungen vereinfacht sich Gleichung (2) zu

$$
v_{a x}=S \cdot n \cdot \zeta
$$

Der in der Praxis sehr komplexe Einfluss des Schüttguts auf die Berechnung der axialen Gutgeschwindigkeit wird dabei nur stark vereinfacht abgebildet. Dies ist insoweit zulässig, da die in der Realität tatsächlich auftretenden Einflüsse durch den empirischen Geschwindigkeitsbeiwert $\zeta$ berücksichtigt werden.

Damit sind nun sowohl die durchflossene Querschnittsfläche als auch die durchschnittliche Geschwindigkeit in Förderrichtung bekannt und der Volumenstrom im geneigten Schneckenförderer lässt sich wie folgt berechnen:

$$
I_{V}=A \cdot v_{a x}=\pi \cdot \frac{1}{4}\left(D^{2}-d^{2}\right) \cdot \varphi \cdot S \cdot n \cdot \zeta
$$

Bis auf den Geschwindigkeitsbeiwert $\zeta$ stehen in Gleichung (5) alle Einflussparameter als Eingangsgrößen bereits fest. Der Geschwindigkeitsbeiwert muss somit als letzter fehlender Parameter noch bestimmt werden, um den Volumenstrom eines Schneckenförderers einfach handhabbar berechnen zu können. Die Bestimmung des Geschwindigkeitsbeiwerts als empirische Kenngröße ist im Folgenden beschrieben.

\section{Datenerhebung}

Zur Erhebung der für die Bestimmung des empirischen Geschwindigkeitsbeiwerts nötigen Daten werden verschiedene Methoden angewandt: Die Auswertung von analytischen Berechnungsverfahren aus früheren Forschungsarbeiten, experimentelle Versuche mit einer Großversuchsanlage für Schneckenförderer sowie Simulationen nach der Diskreten Elemente Methode. Durch diesen Einsatz von verschiedenen Methoden zur Datengewinnung ist es möglich Datensätze für alle (Rand-)Bereiche des Anwendungsbereichs zu gewinnen.

Die experimentellen Versuche werden mit der Großversuchsanlage für Schneckenförderer am Lehrstuhl für Fördertechnik Materialfluss Logistik durchgeführt, dargestellt in Abbildung 3. Diese gleicht sowohl in ihren geometrischen Abmessungen als auch in ihren technischen Daten mehr einer industriellen Förderanlage als einer modellhaften Versuchsanlage. Mit den installierten Schneckenförderern lässt sich ein maximaler Volumenstrom von $I_{V}=0,0275 \mathrm{~m}^{3} / \mathrm{s}$ realisieren. Durch die förder- und messtechnische Ausstattung können die Einflussfaktoren Schneckendrehzahl, Füllungsgrad und Neigung direkt eingestellt oder kontinuierlich überwacht werden. Die erforderliche Antriebsleistung sowie der erzielte Massenstrom lassen sich direkt messen. Aus den aufgezeichneten Daten lässt sich der zur Erstellung der Berechnungsverfahren nötige ermittelte Geschwindigkeitsbeiwert $\zeta^{*}$ berechnen. 


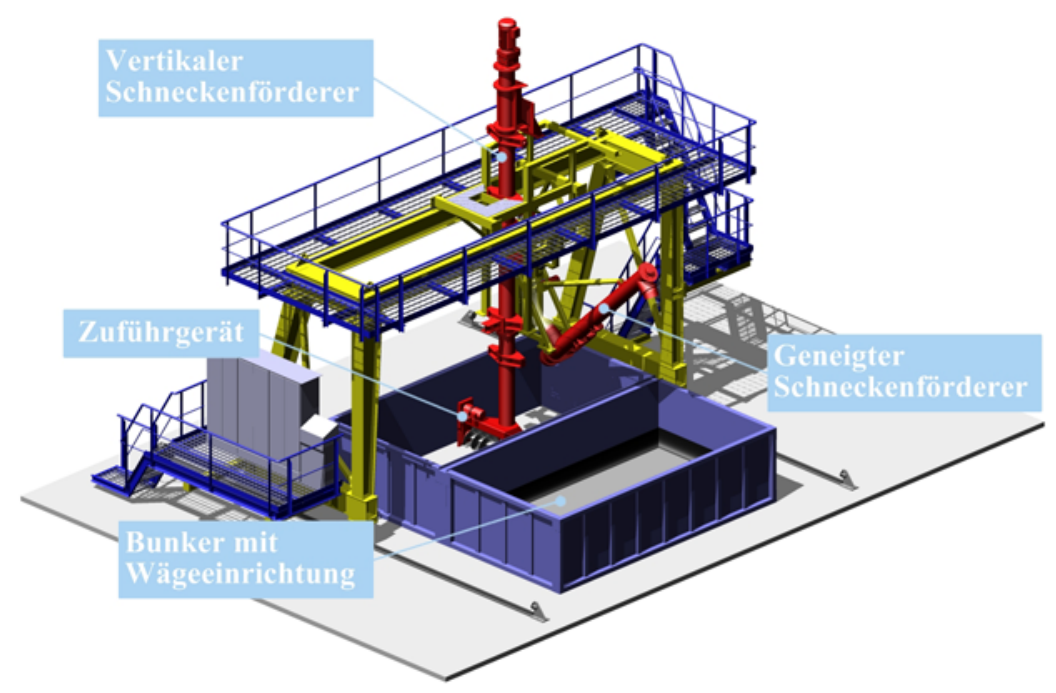

Abbildung 3: Großversuchsanlage für Schneckenförderer

Des Weiteren werden Daten durch Simulationen nach der Diskreten Elemente Methode (DEM) gewonnen. Die Simulationen werden mit einem PET-Granulat als Schüttgut durchgeführt. Dieses Granulat wird auch am realen Großversuchsanlage des Lehrstuhls verwendet und ist somit in den Eigenschaften und im Verhalten bekannt. Da die Simulationen aufgrund der zu erwartenden Rechenzeit nur mit vergrößerten Partikeln durchgeführt werden können, muss das simulierte Schüttgut kalibriert werden. Dazu werden Versuche zur Bestimmung von Schüttguteigenschaften real durchgeführt und in der Simulation nachgebildet. Als Versuche werden die Bestimmung der Schüttdichte, des Böschungswinkels und der Wandreibung gewählt. Der Versuchsaufbau als auch die Simulationsmodelle für die Kalibrierung orientieren sich an den Empfehlungen der FEM 2481 [FEM 2.181]. Zur Kalibrierung werden die Simulationsparameter dabei iterativ so lange verändert bis das Verhalten der Partikel im Simulationsmodell dem realen Schüttgutverhalten mit ausreichender Genauigkeit entspricht. Neben dem simulierten Schüttgut ist natürlich der Förderer an sich von elementarer Bedeutung. Die Maße der Förderer werden zunächst analog zum existierenden Versuchsstand gewählt. Dadurch kann das Simulationsmodell mit Daten der Versuchsanlage verifiziert werden. Sämtliche Geometriemodelle können über die CAD-Daten-Schnittstelle direkt in das Simulationsmodell geladen werden. Das simulierte Modell ist in Abbildung 4 dargestellt. Simuliert wird die Förderung im Schneckenförderer bei verschiedenen Neigungen, Drehzahlen, Füllungsgraden und Schneckendurchmessern. Als Zielgrößen wird die durchschnittliche axiale Gutgeschwindigkeit ausgewertet, um daraus den ermittelten Geschwindigkeitsbeiwert $\zeta^{*}$ berechnen zu können.

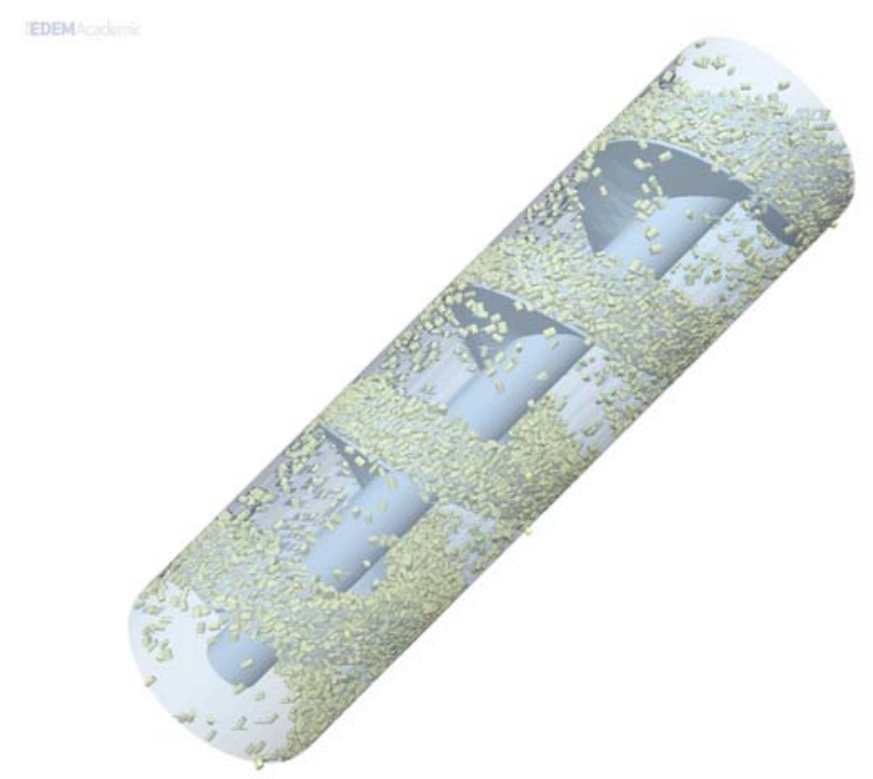

Abbildung 4: Simulationsmodell des Schneckenförderers 
Als dritte Möglichkeit der Datengewinnung wird auf das analytische Berechnungsmodell eines Vorgängerprojekts [Vol-2000] zurückgegriffen, da hiermit gute Ergebnisse für die Bestimmung des Volumenstroms für geneigte Schneckenförderer erzielt werden können, auch wenn die Berechnung sehr aufwendig und nur numerisch möglich ist. Dennoch erscheint es sinnvoll bei Parameterstufen, für die Versuche nicht möglich oder Simulationen zu aufwendig sind, auf diese Berechnungsalgorithmen zurückzugreifen. Zusätzlich werden ebenfalls Parameterkombinationen berechnet, die auch in den Simulationen und Versuchen verwendet werden, um eine Validierung des Verfahrens durchzuführen. Das Berechnungsverfahren liefert als Ergebnis die Gutwinkelgeschwindigkeit des Schüttguts im Schneckenförderer. Daraus kann wieder der ermittelte Geschwindigkeitsbeiwert $\zeta^{*}$ berechnet werden.

Insgesamt können durch diese beschriebenen Methoden die zur Bestimmung des Dimensionierungs- und Auslegungsverfahrens benötigten Kennwerte bestimmt werden. Als unabhängige Einflussgrößen wurden die folgenden Parameter untersucht:

- Schneckendrehzahl $n$ für $21 / \mathrm{s} \leq n \leq 131 / \mathrm{s}$

- Schneckenneigung $\beta$ für $20^{\circ} \leq \beta \leq 90^{\circ}$

- Füllungsgrad $\varphi$ für $0,2 \leq \varphi \leq 0,7$

- Schneckendurchmesser $D$ für $0,2 \mathrm{~m} \leq D \leq 0,8 \mathrm{~m}$

\section{Bestimmung des Geschwindigkeitsbeiwerts}

Als erster Schritt zur Bestimmung des Geschwindigkeitsbeiwerts wird zunächst deskriptiv der Einfluss der Parameter Schneckendrehzahl $n$, Schneckenneigung $\beta$, Schneckendurchmesser $D$ und Füllungsgrad $\varphi$ auf den ermittelten Geschwindigkeitsbeiwert $\zeta^{*}$ untersucht. Da die Darstellung der einzelnen Datenpunkte oft nicht ausreicht um verwertbare Rückschlüsse auf den Einfluss eines Parameters treffen zu können, wird eine lokal gewichtete Regression verwendet. Bei dieser werden die Zusammenhänge zwischen Parametern und Zielgröße völlig flexibel und unabhängig von Restriktionen geschätzt. Es liefert daher sehr genaue Ergebnisse, die aber mit einer hohen Komplexität des Modells erkauft werden müssen: Durch die vollkommen freie Gestaltung der $\mathrm{Zu}-$ sammenhänge ist es meist nicht mehr möglich den gefundenen Zusammenhang in kompakten Formeln, wie sie im Rahmen dieses Projekts gewünscht sind, darzustellen. Dennoch erlaubt die grafische Darstellung des modellierten Zusammenhangs einen Blick auf den eventuell zugrundeliegenden Effekt. In Abbildung 5 sind die Ergebnisse jeweils in Abhängigkeit der einzelnen Einflussparameter dargestellt. Die unterschiedlichen Einflüsse auf den Geschwindigkeitsbeiwert sind in den einzelnen Diagrammen zu erkennen.
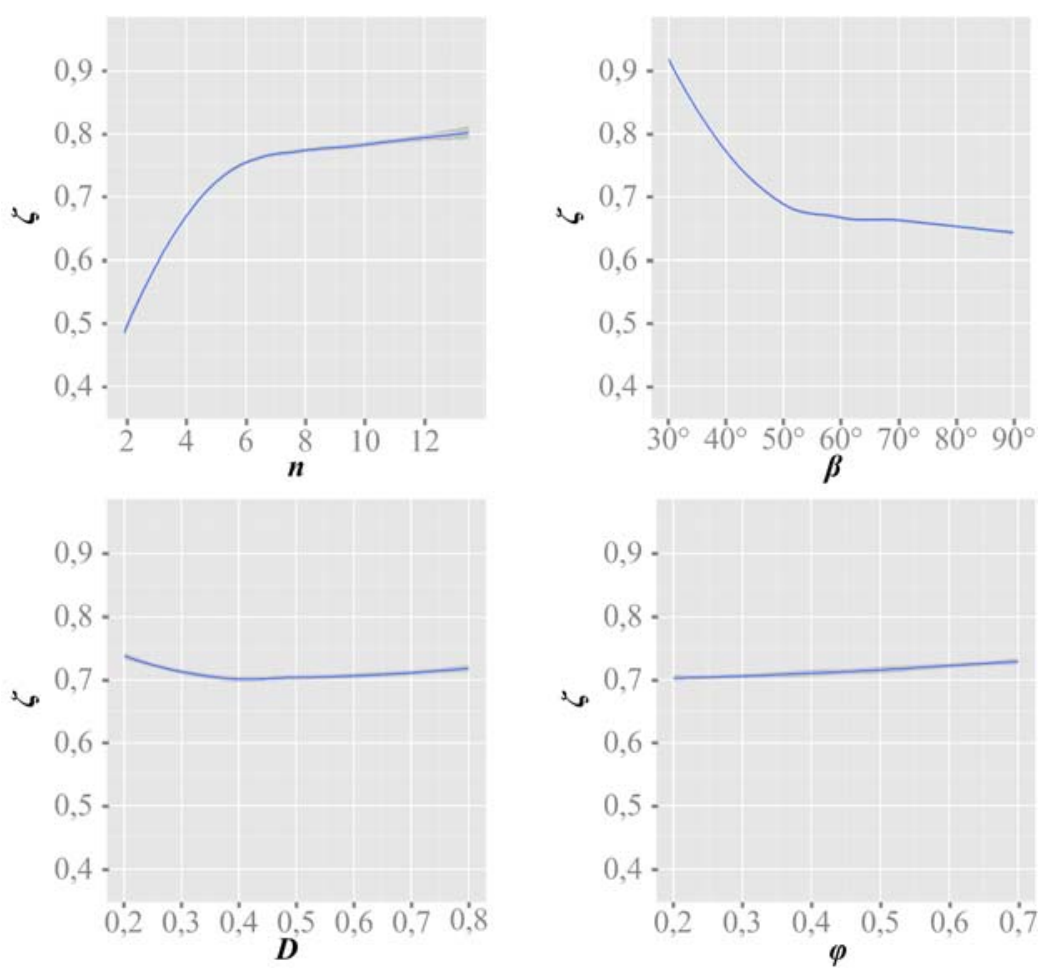

Abbildung 5: Ergebnisse der lokal gewichteten Regression 
Im Diagramm links oben ist der ermittelte Geschwindigkeitsbeiwert $\zeta^{*}$ über der Schneckendrehzahl $n$ aufgetragen. Zu erkennen ist ein zunächst starker positiver Zusammenhang, der sich ab Schneckendrehzahlen von ca. $n=6$ 1/s deutlich abschwächt. Der Verlauf der Kurve entspricht einer Logarithmus- oder Potenzfunktion. Der Einfluss der Schneckenneigung $\beta$ ist oben rechts dargestellt. Hier lässt sich eine reziproke Proportionalität oder ebenfalls eine Potenzfunktion identifizieren. Die Einflüsse von Füllungsgrad $\varphi$ und Schneckendurchmesser $D$, abgebildet unten links und rechts, sind augenscheinlich am Schwächsten. Für den Füllungsgrad lässt sich ein leichter linearer Einfluss, für den Schneckendurchmesser ein leicht nichtlinearer Einfluss erkennen.

Diese identifizierten Zusammenhänge bilden die Grundlage für das Regressionsmodell für den Geschwindigkeitsbeiwert. Erste Regressionsrechnungen zeigen, dass der Einfluss der Schneckendrehzahl $n$ durch eine Potenzfunktion sehr gut angenähert werden kann. Für den Einfluss der Schneckenneigung $\beta$ hat sich eine Potenzfunktion als beste Alternative ergeben. Für den Einfluss des Schneckendurchmessers $D$ kann mit ausreichender Genauigkeit ein linearer Zusammenhang angenommen werden, um das Modell zu vereinfachen. Weiter zeigte sich, dass sich der Einfluss der Schneckenneigung nicht nur auf den Geschwindigkeitsbeiwert sondern auch auf die Einflüsse der anderen Parameter auswirkt. Dies wurde bei der folgenden Bildung des Regressionsmodells berücksichtigt. Dieses hat die folgende Form:

$$
\zeta=c_{1}+c_{2} \cdot \beta^{c_{3}}+c_{4} \cdot n^{c_{5}}+c_{6} \cdot D+c_{7} \cdot \varphi+\left(c_{8} \cdot n^{c_{5}}+c_{9} \cdot D+c_{10} \cdot \varphi\right) \cdot \beta^{c_{11}}
$$

Mit Hilfe eines Gauß-Newton-Algorithmus werden die Koeffizienten $c_{k}, k=1, \ldots, 11$, geschätzt. Die resultierenden Schätzungen sind in Tabelle 1 dargestellt. Die Wurzel des mittleren quadratischen Fehlers des Modells, also die durchschnittliche Abweichung des ermittelten vom berechneten Wert, beträgt $\sqrt{M Q F}=0,0125$. Dies entspricht auch bei den kleinsten ermittelten Geschwindigkeitsbeiwerten $\zeta^{*}$ einer durchschnittlichen Abweichung von kleiner 3\% und zeigt die ausreichende Genauigkeit des berechneten Regressionsmodells.

Tabelle 1: Geschätzte Koeffizienten für das Regressionsmodell

\begin{tabular}{|c|c|c|c|}
\hline Koeffizient & Schätzung & Koeffizient & Schätzung \\
\hline$c_{1}$ & 0,782768 & $\mathrm{c}_{2}$ & 983,0707 \\
\hline$c_{3}$ & $-2,532883$ & $\mathrm{c}_{4}$ & $-1,191981$ \\
\hline$c_{5}$ & $-0,799694$ & $\mathrm{c}_{6}$ & 0,282653 \\
\hline$c_{7}$ & 0,099595 & $\mathrm{c}_{8}$ & $472.364,1$ \\
\hline $\mathrm{c}_{9}$ & $-112.625,1$ & $\mathrm{c}_{10}$ & $40.349,4$ \\
\hline
\end{tabular}

Um abschließend die Güte des Regressionsmodells beurteilen zu können, werden die durch das Regressionsmodell berechneten Werte für den Geschwindigkeitsbeiwert $\zeta$ mit den ermittelten Werten $\zeta^{*}$ verglichen und zunächst grafisch dargestellt. Je näher die Punkte in Abbildung 6 auf der Winkelhalbierenden liegen, desto besser ist dabei das Modell.

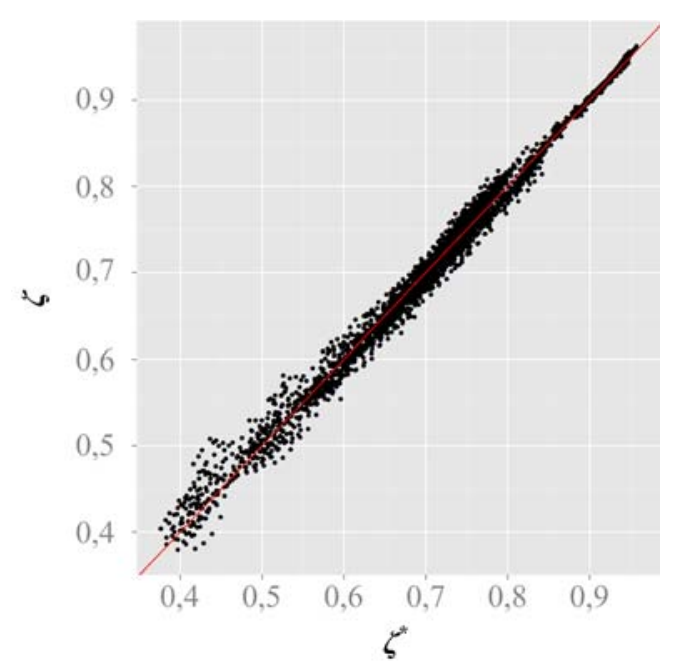

Abbildung 6: Modellanpassung für den Geschwindigkeitsbeiwert 
Um bewerten zu können, wo die Abweichungen des Modells liegen, sind in Abbildung 7 die ermittelten und geschätzten Werte des Geschwindigkeitsbeiwerts in den Kategorien Schneckendrehzahl, Füllungsgrad und Schneckendurchmesser für die Schneckenneigung $\beta=60^{\circ}$ beispielhaft dargestellt. Für die anderen untersuchten Schneckenneigungen ergeben sich sehr ähnliche Ergebnisse. Zu erkennen ist, dass in nahezu allen Feldern eine gute Übereinstimmung der ermittelten Datenpunkte mit dem berechneten Modell (Kurve) vorhanden ist. Einzig in Randbereichen kleiner Füllungsgrade und Schneckendurchmesser gibt es kleinere Abweichungen.

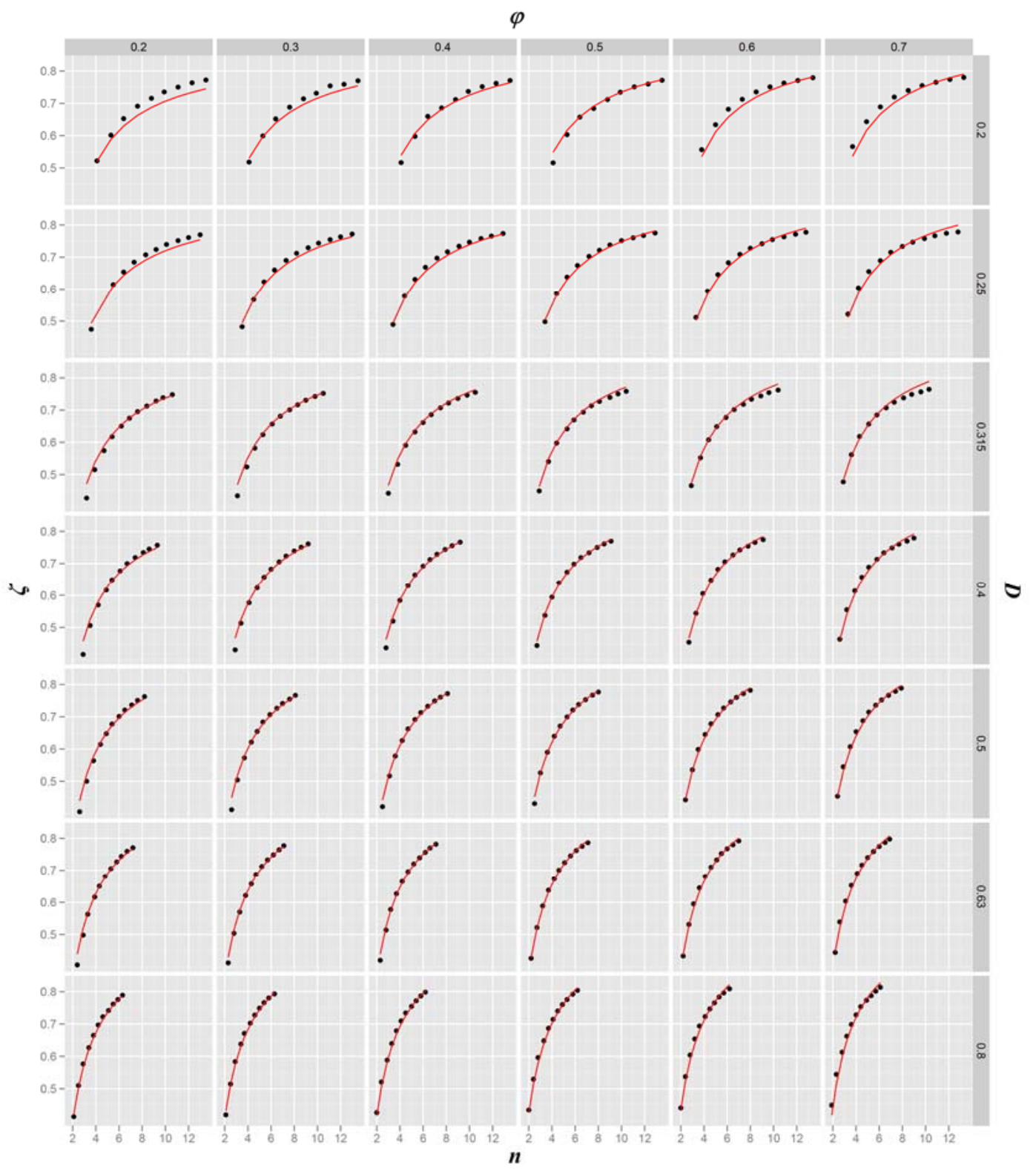

Abbildung 7: Modellanpassung nach Kategorien

Das gefundene Regressionsmodell ist also zur Berechnung des Geschwindigkeitsbeiwerts geeignet. Der erzielbare Volumenstrom $I_{V}$ eines geneigten Schneckenförderers kann damit durch die Gleichungen (5) und (6) einfach und zuverlässig berechnet werden. 


\section{Zusammenfassung}

Im durchgeführten Projekt wird die Förderung von Schüttgut in Schneckenförderern im Neigungsbereich zwischen $20^{\circ}$ und $60^{\circ}$ untersucht. Ziel ist die Entwicklung eines einfachen, praxistauglichen und sicheren Verfahren zur Berechnung des erzielbaren Volumenstroms. Wie auch für andere Betriebsbereiche von Schneckenförderern (horizontal bis leicht geneigt und vertikal), wird bei der Bestimmung der Berechnungsvorschriften ein semiempirisches Verfahren angewendet, um die komplexen Vorgänge im Inneren des Schneckenförderers, die analytisch nicht beschreibbar sind, abbilden zu können. Grundlage für ein solches Verfahren ist ein analytischer Grundansatz, der um empirische Kenngrößen erweitert wird. Diese bilden darin die analytisch nicht erfassbaren Einflüsse in Abhängigkeit der bekannten Parameter ab. Als Grundansatz für die betrachtete Zielgröße Volumenstrom wird die aus der DIN 15262 bekannte Formel in leicht modifizierter Form verwendet.

Um die empirischen Kenngrößen zuverlässig für den gesamten Anwendungsbereich bestimmen zu können, ist eine ausreichende Menge an Datensätzen nötig. Diese notwendigen Datensätze werden mit drei verschiedenen Möglichkeiten erzeugt: Experimentelle Untersuchungen an einer Großversuchsanlage, Simulationen nach der Diskreten Elemente Methode und Berechnung mit numerischen Verfahren, die in Vorgängerprojekten erarbeitet wurden. Durch diese Aufspaltung der Datengewinnung auf verschiedene Methoden ist es möglich alle Bereiche des Anwendungsbereichs mit Datensätzen zu belegen.

Die gewonnen Datensätze werden mit statistischen Methoden eingehend ausgewertet. In einem ersten Schritt wird dazu deskriptiv untersucht, welche Parameter Einfluss auf die bestimmten Kennwerte haben und welcher Art diese Einflüsse sind. Aufbauend darauf können in einem zweiten Schritt diese Erkenntnisse in die Regressionsmodelle für die empirische Kenngröße einfließen. Die berechnete Regressionsgleichung wird auf Ihre Güte hin untersucht, um sicherzustellen, dass die Ergebnisse die Anforderungen an die Genauigkeit erfüllen. Ergebnis sind einfache Formeln, die die Zusammenhänge der Einflussparameter auf die Kenngrößen beschreiben und damit eine zuverlässige Vorhersage ermöglichen. Zusammen mit dem analytischen Grundansatz kann damit der mögliche Volumenstrom und die dazu nötige Antriebsleistung berechnet werden.

\section{Formelzeichen}

\begin{tabular}{ccl} 
Formelzeichen & Einheit & Name \\
\hline$A$ & {$\left[\mathrm{~m}^{2}\right]$} & Querschnittsfläche \\
$D$ & {$[\mathrm{~m}]$} & Schneckendurchmesser \\
$I_{V}$ & {$\left[\mathrm{~m}^{3} / \mathrm{s}\right]$} & Volumenstrom \\
$M Q F$ & {$[-]$} & Mittlerer quadratischer Fehler \\
$S$ & {$[\mathrm{~m}]$} & Schneckenganghöhe \\
$c$ & {$[-]$} & Regressionskoeffizient \\
$d$ & {$[\mathrm{~m}]$} & Wellendurchmesser \\
$n$ & {$[1 / \mathrm{s}]$} & Schneckendrehzahl \\
$k$ & {$[-]$} & Laufvariable \\
$v_{a x}$ & {$[\mathrm{~m} / \mathrm{s}]$} & Gutaxialgeschwindigkeit \\
$\beta$ & {$\left[{ }^{\circ}\right]$} & Schneckenneigung \\
$\zeta$ & {$[-]$} & Geschwindigkeitsbeiwert \\
$\zeta^{*}$ & {$[-]$} & Ermittelter Geschwindigkeitsbeiwert \\
$\varphi$ & {$[-]$} & Füllungsgrad \\
$\omega_{G}$ & {$[1 / \mathrm{s}]$} & Gutwinkelgeschwindigkeit \\
$\omega_{S}$ & {$[1 / \mathrm{s}]$} & Schneckenwinkelgeschwindigkeit
\end{tabular}




\section{Literatur}

[Blo-2008] Blomeyer, N.: Erweiterung und Verifikation von Auslegungsverfahren für HochleistungsSchneckenförderer für Schüttgut; Dissertation, München, Technische Universität München, 2008

[Böt-1963] Böttcher, S.: Eine allgemeine Analyse der Aufwärtsförderung eines Einzelkörpers in Schneckenförderern beliebiger Neigung, Tl. 1 - 3; In: VDI-Zeitschrift, Jahrgang 105 (1963), Nr. 14, S. 581-593; Nr. 16, S. 663-671; Nr. 18, S. 743-754

[DIN 15262] DIN 15262:1983-01: Stetigförderer - Schneckenförderer für Schüttgut - Berechnungsgrundsätze, Deutsches Institut für Normung e.V.

[FEM 2.181] FEM 2.181:1989: Spezifische Schüttguteigenschaften bei der mechanischen Förderung, Fédération Européenne de la Manutention

[Gab-1981] Gabler, H.: Theoretische und experimentelle Untersuchung der Förderung in steilen und senkrechten Schneckenförderern; Dissertation, München, Technische Universität München, 1981

[Gün-2002] Günthner, W. A.; Fottner, J.; Rong, O.: Empirische Entwicklung von Kenngrößen zur Auslegung von Hochleistungs-Schneckenförderern für Schüttgut; Herbert Utz Verlag, München, 2002

[Gün-2006] Günthner, W. A.; Blomeyer, N.; Rong, O.: Entwicklung von praxistauglichen Auslegungsverfahren für Schüttgut-Hochleistungs-Schneckenförderer, Forschungsbericht, Lehrstuhl für Fördertechnik Materialfluss Logistik, Technische Universität München, Garching bei München, 2006

[Ron-2004] Rong, O.: Auslegungsverfahren für Hochleistungs-Schneckenförderer; Dissertation, München, Technische Universität München, 2004

[VDI 2330] VDI 2330:1993-02: Schneckenförderer, Verein deutscher Ingenieure e.V.

[Vol-2000] Vollmann, A.: Untersuchung der Schüttgutförderung in geneigten Schneckenförderern; Dissertation, München, Technische Universität München, 2000 